\title{
Effects of alcohol on intestinal epithelial barrier permeability and expression of tight junction-associated proteins
}

\author{
YING WANG, JING TONG, BING CHANG, BAIFANG WANG, DAI ZHANG and BINGYUAN WANG \\ Department of Gastroenterology, The First Affiliated Hospital of China Medical University, \\ Shenyang, Liaoning 110001, P.R. China
}

Received July 12, 2013; Accepted February 27, 2014

DOI: $10.3892 / \mathrm{mmr} .2014 .2126$

\begin{abstract}
The present study aimed to investigate the effects of alcohol on intestinal epithelial barrier permeability and expression of the tight junction-associated proteins, zonula occludens-1 (ZO-1) and claudin-1. An alcohol-treated Caco-2 intestinal epithelial cell monolayer in vitro model was used to investigate whether alcohol is able to induce intestinal barrier dysfunction and decrease expression of ZO-1 and claudin-1. MTT assay results revealed unaltered cell viability at alcohol concentrations of $<5 \%$. Caco- 2 cells in the $5 \%$ alcohol-treated groups exhibited a significant time-dependent decrease in transepithelial electrical resistance (TEER) and an increase in fluorescent yellow flux rate compared with the control cells. ZO-1 expression exhibited a progressive decline following $20 \mathrm{~min}$ of incubation, reached its minimum levels at $60 \mathrm{~min}$ and then showed an increasing trend following $60 \mathrm{~min}$ of incubation. In addition, claudin-1 expression exhibited a progressive increase following $60 \mathrm{~min}$ of incubation, reached its maximum levels at $60 \mathrm{~min}$ and then showed an increasing trend following $60 \mathrm{~min}$ of incubation. Alterations in the expression of the ZO-1 and claudin-1 proteins revealed trends consistent with changes in the TEER value and the fluorescent yellow transmittance rate in the Caco- 2 cells. The results of this study indicate that alcohol is able to increase the intestinal epithelial barrier permeability in a dose- and time-dependent manner. Alcohol induces a change in the expression of the tight junction-associated proteins, ZO-1 and claudin-1, which are two major sites of alcohol action, thus increasing intestinal epithelial barrier permeability.
\end{abstract}

Correspondence to: Professor Bingyuan Wang, Department of Gastroenterology, The First Affiliated Hospital of China Medical University, 155 Nanjing Street, Heping, Shenyang, Liaoning 110001, P.R. China

E-mail: cmulh_wby@126.com

Abbreviations: ALD, alcoholic liver disease; ZO-1, zonula occludens-1; TEER, transepithelial electrical resistance; LDH, lactate dehydrogenase

Key words: alcohol, intestinal epithelial barrier, permeability, tight junction-associated protein

\section{Introduction}

Alcoholic liver disease (ALD) is a major cause of liver-associated morbidity and mortality worldwide (1). ALD encompasses a spectrum of conditions, ranging from simple steatosis to chronic hepatitis with fibrosis or cirrhosis (2). Although the exact mechanisms of the pathogenesis of ALD are not fully understood, is has been suggested that tight-junction protein expression and intestinal barrier function have crucial roles in the development of ALD (3). Previous studies have indicated that alcohol increases intestinal permeability to macromolecules and leads to an abnormal leakage of bacterial endotoxins, thereby inducing alcohol-induced liver injury $(4,5)$. Numerous other studies have also indicated that chronic alcohol consumption may accelerate the progression of ALD via oxidative stress, increased intestinal permeability and an elevated risk of endotoxemia $(6,7)$. It is now generally accepted that damaged intestinal epithelial integrity and intestinal barrier dysfunction are the two fundamental causes of increased intestinal permeability $(8,9)$. Therefore, an improved understanding of the mechanisms involved in increased intestinal permeability induced by alcohol may lead to the development of more effective prevention and treatment strategies for ALD (10).

The intestinal epithelial barrier is mainly composed of a monolayer of cells with intercellular tight junctions, a complex three-dimensional structure and a thick mucosal gel layer secreted by the mucous membrane; it also provides a dynamic and regulated barrier to the extracellular flux of the lumina $(11,12)$. Tight junctions are essential in maintaining intestinal mucosal integrity, which is able to effectively prevent bacteria, endotoxins and other harmful substances from entering the blood stream through the intestinal barrier (13). Previous studies have indicated that altered expression of the tight junction-associated proteins, zonula occludens-1 (ZO-l) and claudin-1, is associated with increased intestinal permeability and a higher susceptibility to ALD $(14,15)$. However, no previous study investigating whether alcohol is able to induce intestinal epithelial barrier dysfunction and affect the expression of these tight junction-associated proteins is available. The present study used an alcohol-treated Caco-2 intestinal epithelial cell monolayer in vitro model to observe the effects of alcohol on intestinal epithelial barrier permeability and the expression of tight junction-associated proteins. 


\section{Materials and methods}

Culture of Caco-2 cells. The human colon adenocarcinoma Caco-2 cell line was obtained from the American Type Culture Collection (ATCC; Rockville, MD, USA). Caco-2 cells were cultured in Dulbecco's modified Eagle's medium (DMEM; Gibco-BRL, Grand Island, NY, USA) supplemented with $0.1 \mathrm{mmol} / 1$ non-essential amino acids, $10 \mathrm{mmol} / 1 \mathrm{HEPES}$, $4.5 \mathrm{mg} / \mathrm{ml}$ glucose, $100 \mathrm{U} / \mathrm{ml}$ penicillin, $100 \mathrm{U} / \mathrm{ml}$ streptomycin, $4 \mathrm{mM}$ glutamine and $10 \%$ fetal bovine serum (FBS; Hyclone, Logan, UT, USA), and incubated in a humidified atmosphere $\left(95 \%\right.$ air, $5 \% \mathrm{CO}_{2}$ ) at $37^{\circ} \mathrm{C}$.

Establishment of an in vitro model of the intestinal epithelial cell barrier. To establish an in vitro model of the intestinal epithelial barrier, the Caco-2 cells were plated on Transwell filters (Corning, Inc., Corning, NY, USA) and regularly monitored visually using an inverted microscope (Olympus, Tokyo, Japan) and through epithelial resistance measurements.

Epithelial resistance measurement in Ussing chambers. Firstly, the abdomen was carefully incised from the midline and a 1- to $1.5-\mathrm{cm}$ sample of the small intestine was dissected out. Then, an exposed area of $0.126 \mathrm{~cm}^{2}$ was utilized to mount the sample vertically in Ussing chambers. Following that, Krebs-Ringer bicarbonate solution (in $\mathrm{mM}: 128 \mathrm{NaCl}$, $5.1 \mathrm{KCl}, 1.4 \mathrm{CaCl}_{2}, 1.3 \mathrm{MgCl}_{2}, 21 \mathrm{NaHCO}_{3}, 1.3 \mathrm{KH}_{2} \mathrm{PO}_{4}$ and $10 \mathrm{NaH}_{2} \mathrm{PO}_{4}$, pH 7.4) gassed with $95 \% \mathrm{O} 2-5 \% \mathrm{CO}_{2}$ was used to bath the tissues. Following a 15 -min equilibration period, an EVC 4000 Precision V/I clamp device (World Precision Instruments, Sarasota, FL, USA) was used for the measurement of the transepithelial electrical voltage and current per $5 \mathrm{~min}$ until $30 \mathrm{~min}$. The calculation of epithelial resistance was achieved using Ohm's law $(\mathrm{R}=\mathrm{V} / \mathrm{I})$. The experiment was repeated at least three times using a different tissue sample each time.

Viability assay. The Caco-2 cells were seeded on flat-bottomed, 96-well tissue culture plates. Following incubation with alcohol $(1,2.5,5,7.5$ and $10 \%)$ for $4 \mathrm{~h}$, the cells were incubated with $100 \mu \mathrm{l}$ MTT [5 $\mathrm{mg} / \mathrm{ml}$ solution in phosphate-buffered saline (PBS); Sigma-Aldrich, Taufkirchen, Germany] for $1 \mathrm{~h}$ at $37^{\circ} \mathrm{C}$. The culture media was removed, and $150 \mu \mathrm{l}$ dimethylsulfoxide was added to each well. The absorbance of the resulting colored solution was measured at $570 \mathrm{~nm}$ with a microplate reader (Wallac Victor 2; Perkin-Elmer, Waltham, MA, USA). In this assay, the yellow MTT solution is converted into a blue formazan dye within the mitochondria and deposited intracellularly. The intensity of the blue stain is then quantitatively assessed by spectrometry and used as a measure of cell viability.

Cell viability was also assessed by measuring the release of cytosolic enzymes. The electrical resistance of Caco-2 cell monolayers cultured on Transwell filters was assessed using a Millicell-ERS instrument (Millipore, Bedford, MA, USA). The electrical resistance was expressed in units of $\Omega \cdot \mathrm{cm}^{2}$ using the surface area of the Transwell insert.

Fluorescent yellow $(40 \mu \mathrm{g} / \mathrm{ml}$; Sigma-Aldrich) in serum-free DMEM was added to the upper chamber of the Transwell system. Following incubation with alcohol for 0, 20,
$40,60,120$ or $180 \mathrm{~min}$, the media from the lower chamber was collected. The absorbance was assessed using a fluorescence spectrophotometer (excitation wavelength, $427 \mathrm{~nm}$; emission wavelength, $536 \mathrm{~nm}$ ), and the concentration of fluorescent yellow was calculated based on the standard curve. The fluorescent yellow flux rate (\%) was equal to the fluorescent yellow concentration in the lower chamber/the fluorescent yellow concentration in the upper chamber. Lactate dehydrogenase (LDH) was added into the medium. The Caco- 2 cells were incubated with the varying concentrations of alcohol for $4 \mathrm{~h}$. LDH assessment was performed in $250 \mu \mathrm{l}$-aliquots using an LDH kit (Doles reagents, Goiânia, GO, Brazil).

Assessment of intestinal epithelial barrier permeability. Transepithelial electrical resistance (TEER) and the fluorescent yellow flux rate were assessed to estimate the effects of alcohol on the paracellular permeability in the Caco-2 cell monolayers. The electrical resistance of the Caco- 2 cell monolayers cultured on Transwell filters was assessed using a Millicell-ERS instrument (Millipore, Bedford, MA, USA). The electrical resistance was expressed in units of $\Omega \bullet \mathrm{cm}^{2}$ using the surface area of the Transwell insert. Fluorescent yellow ( $40 \mu \mathrm{g} / \mathrm{ml}$; Sigma-Aldrich) in serum-free DMEM was added to the upper chamber of the Transwell system. Following incubation with alcohol for the varying times indicated, the media from the lower chamber was collected. The absorbance was assessed using a fluorescence spectrophotometer (excitation wavelength, $427 \mathrm{~nm}$; emission wavelength, $536 \mathrm{~nm}$ ), and the fluorescent yellow concentration was calculated based on the standard curve. The fluorescent yellow flux rate (\%) was equal to the fluorescent yellow concentration in the lower chamber/the fluorescent yellow concentration in the upper chamber.

Western blot analysis. The Caco-2 cells were washed with Dulbecco's PBS (D-PBS) containing $0.1 \mathrm{mM}$ ethylenediamine tetraacetic acid (EDTA) three times without calcium and magnesium. The Caco-2 cells were then homogenized in $1 \mathrm{ml}$ lysis buffer A [(Shanghai Pik-day, Shanghai, China) $2 \mathrm{mM}$ EDTA, $10 \mathrm{mM}$ ethylene glycol tetraacetic acid, $0.4 \% \mathrm{NaF}, 20 \mathrm{mM}$ Tris-HCl, protease inhibitor cocktail, phosphatase inhibitor and $1 \%$ Triton X-100 $(\mathrm{pH} 7.5)]$ at $4^{\circ} \mathrm{C}$. Samples were centrifuged at $14,000 \mathrm{x}$ g for $30 \mathrm{~min}$, and the supernatant was transferred to a separate tube and collected as the soluble fraction. Buffer A (150 $\mu \mathrm{l})$ with $1 \%$ sodium dodecyl sulfate (SDS) at $4^{\circ} \mathrm{C}$ was then added to the pellet. The pellet's structure was disrupted with an ultrasonic crusher. The samples were then centrifuged at $14,000 \mathrm{x}$ g for $30 \mathrm{~min}$ at $4^{\circ} \mathrm{C}$. The supernatant was collected as the insoluble fraction. Equal amounts of protein $(40-50 \mu \mathrm{g})$ were separated by SDS-PAGE and processed for immunoblotting with antibodies for ZO-1 (diluted 1:1,000; Santa Cruz Biotechnology, Inc., Santa Cruz, CA, USA) and claudin-1 (diluted 1:100; Santa Cruz Biotechnology, Inc.). The protein bands were scanned using ChemiImager 5500 V2.03 software (Alpha US Inc., Miami, FL, USA), and the integrated density values (IDVs) were calculated using a computerized image analysis system (Fluor Chen 2.0; Olympus, Yokohama, Japan) and normalized to that of $\beta$-actin.

Immunofluorescence. The Caco-2 cell monolayers grown on glass coverslips were fixed with $4 \%$ paraformaldehyde and 
permeabilized with $0.5 \%$ Triton X-100. Following blocking with $2 \%$ bovine serum albumin in PBS, the cells were incubated with rabbit anti-ZO-1 (diluted 1:50; Santa Cruz Biotechnology, Inc.) and rabbit anti-claudin-1 (diluted 1:100; Zymed, South San Francisco, CA) for visualization of the distribution of ZO-1 and claudin-1. The glass slides were analyzed using immunofluorescence microscopy (Olympus, Tokyo, Japan).

Statistical analysis. Experiments were repeated at least three times. Continuous variables are expressed as the mean \pm standard deviation. Categorical data are presented as frequencies and percentages. Differences between the groups were compared using the two-tailed, non-paired Student's t-test or one-way analysis of variance for continuous variables, where appropriate. Comparisons of categorical variables between the groups were performed using the $\chi^{2}$ test. All tests of statistical significance were two-sided, with $\mathrm{P}<0.05$ being considered to indicate a statistically significant difference. The statistical analyses were performed using SPSS 17.0 (SPSS, Inc., Chicago, IL, USA).

\section{Results}

Effects of alcohol on Caco-2 cell viability. The Caco-2 cells were initially treated for $3 \mathrm{~h}$ with varying concentrations $(1,2.5,5,7.5$ and $10 \%)$ of alcohol, and cell viability was evaluated using the MTT and LDH assays. The MTT assay results showed that the cell viability was not altered at alcohol concentrations of $<5 \%$ (Fig. 1). Furthermore, the LDH assay indicated that alcohol did not increase the release of the cytosolic enzyme LDH into the media at concentrations of $\leq 10 \%$ (Fig. 2). Alcohol concentrations of $>5 \%$ caused cell shedding but not cell fragmentation. At an alcohol concentration of $10 \%$, the ratio of cell shedding was up to $51.67 \pm 3.36 \%$. Therefore, subsequent experiments were performed using alcohol concentrations of $1-5 \%$.

Effects of alcohol on paracellular permeability. As shown in Fig. 3, 5\% alcohol induced a significant time-dependent decrease in transepithelial electrical resistance (TEER), with the lowest value obtained after 60 min of alcohol treatment. A time-dependent increase in the fluorescent yellow flux rate as a result of alcohol treatment was observed, with the maximum flux rate occurring at $60 \mathrm{~min}$ (Fig. 4). The aforementioned results indicated that the decreased TEER value was associated with an increase in the fluorescent yellow flux rate.

Effects of ethanol on the expression of ZO-1. The Caco-2 cells in the alcohol treatment groups were incubated with $5 \%$ alcohol for 0, 20, 40, 60, 120 and $180 \mathrm{~min}$. As shown in Fig. 5, the expression levels of the tight junction-associated protein, ZO-1, exhibited a progressive decline following $20 \mathrm{~min}$ of incubation, reached a minimum level at $60 \mathrm{~min}$ and exhibited an increasing trend after $60 \mathrm{~min}$ of incubation. Significant differences in ZO-1 expression between the control and alcohol groups were observed at 60 min of incubation $(\mathrm{P}<0.05)$.

Effects of alcohol on the expression of claudin-1. Expression of the claudin-1 protein in the alcohol-treated Caco-2 cells showed a progressive increase following $60 \mathrm{~min}$ of incubation, reached its maximum level at $60 \mathrm{~min}$ and then showed a decreasing

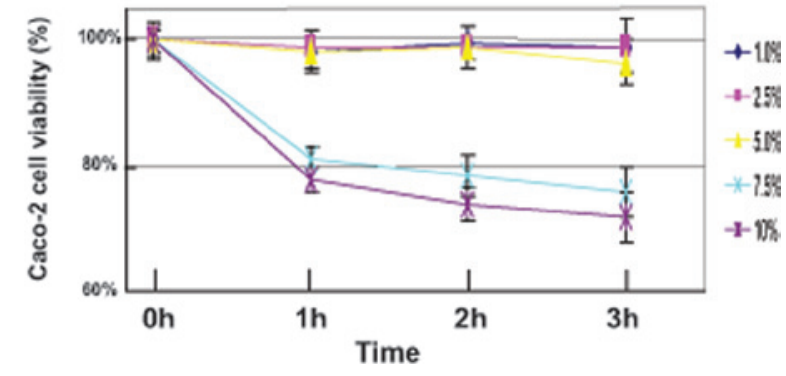

Figure 1. Effect of alcohol on Caco-2 cell viability. The MTT assay results showed that the cell viability was not altered at alcohol concentrations of $<5 \%$.

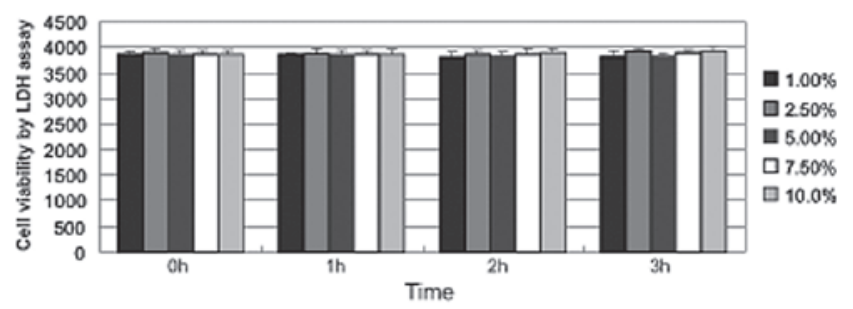

Figure 2. Effect of alcohol on the concentration of the cytosolic enzyme, $\mathrm{LDH}$, in Caco-2 cells. The LDH assay indicated that alcohol did not increase the release of the cytosolic enzyme LDH into the media at concentrations of $\leq 10 \%$. LDH, lactate dehydrogenase.

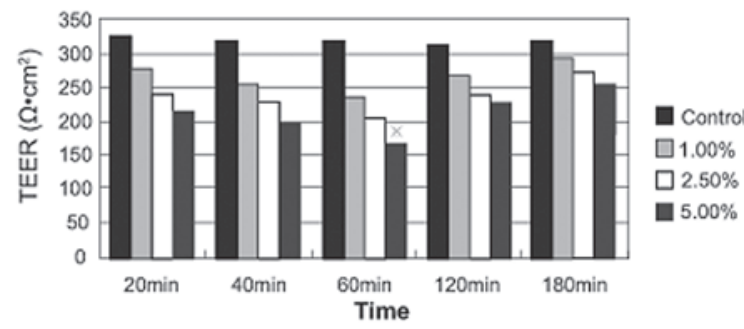

Figure 3. Effect of alcohol on changes in the TEER of the Caco-2 cells Caco- 2 cells in the $5 \%$ alcohol-treated groups exhibited a significant time-dependent decrease in TEER. ${ }^{*} \mathrm{P}<0.05$ compared with control group. TEER, transepithelial electrical resistance.

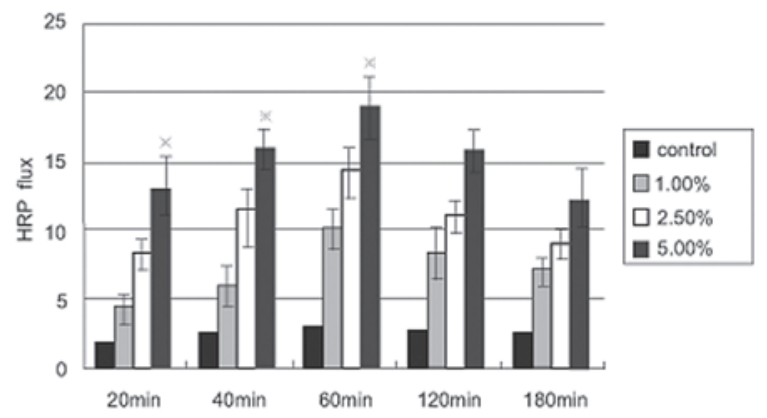

Figure 4. Effect of alcohol on the changes in the fluorescent yellow flux rate of the Caco- 2 cells. Caco-2 cells in the 5\% alcohol-treated groups exhibited an increase in fluorescent yellow flux rate compared with the control cells.

trend following $60 \mathrm{~min}$ of incubation (Fig. 6). Significant differences in claudin- 1 expression were identified among the control and alcohol-treated groups at $60 \mathrm{~min}(\mathrm{P}<0.05)$. 

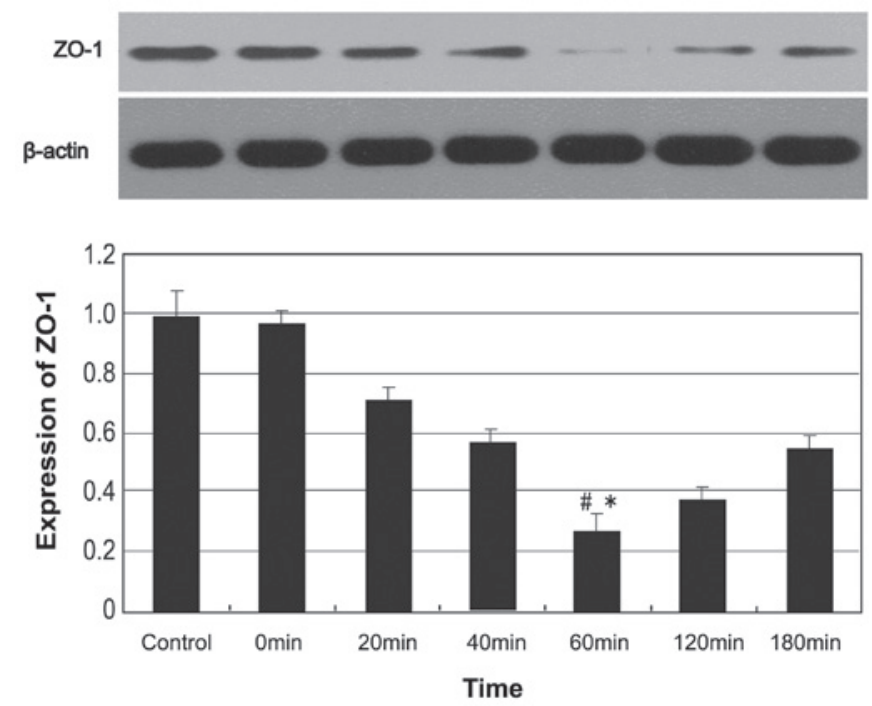

Figure 5. Effect of 5\% alcohol on ZO-1 expression in the Caco-2 cells. Alterations in the expression of the ZO-1 protein revealed trends consistent with changes in the TEER value and the fluorescent yellow transmittance rate in the Caco- 2 cells. ${ }^{*} \mathrm{P}<0.05$ compared with control group; ${ }^{*} \mathrm{P}<0.05$ compared with 0 min. ZO-1, zonula occludens-1.
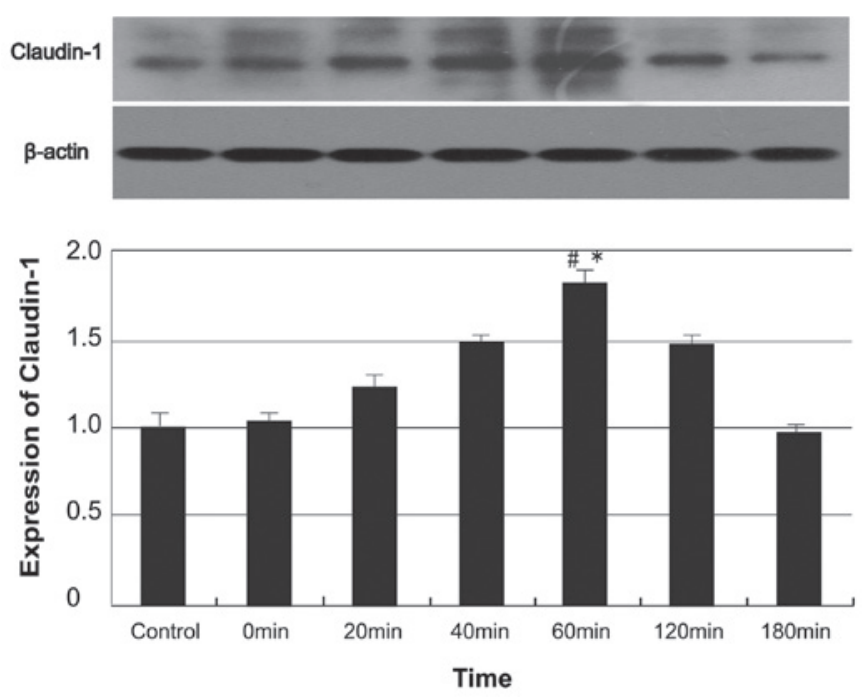

Figure 6. Effect of 5\% alcohol on claudin-1 expression in the Caco-2 cells. Alterations in the expression of the claudin-1 protein revealed trends consistent with changes in the TEER value and the fluorescent yellow transmittance rate in the Caco- 2 cells. $\mathrm{P}<0.05$ compared with control group; ${ }^{*} \mathrm{P}<0.05$ compared with 0 min.

Effects of alcohol on the distribution of ZO-1 and claudin-1. The distribution of ZO-1 and claudin-1 was assessed using immunofluorescence microscopy (Fig. 7). Alcohol was shown to induce the discontinuous distribution of ZO-1 and claudin-1 at the cellular membrane.

\section{Discussion}

Experimental and clinical studies have revealed evidence that damaged intestinal epithelial integrity and intestinal barrier dysfunction are the two fundamental causes of increased intestinal permeability $(8,9)$. Intestinal epithelial barrier integrity
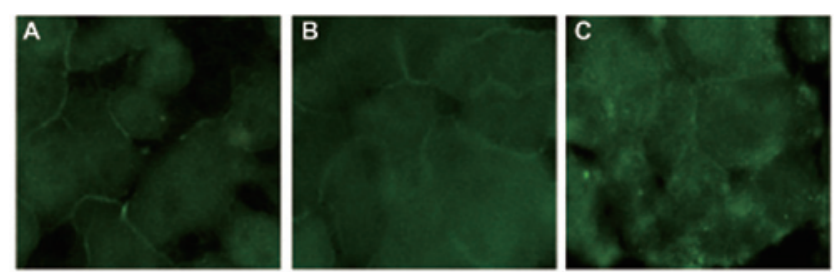

Figure 7. Immunofluoresence localization (magnification, $\mathrm{x} 400$ ) of ZO-1 and claudin-1 proteins in the Caco-2 cells. (A) Control; (B) Caco-2 cells treated with ethanol (5\%) for $20 \mathrm{~min}$; (C) Caco-2 cells treated with ethanol (5\%) for $60 \mathrm{~min}$. Alcohol was shown to induce the discontinuous distribution of ZO-1 and claudin-1 at the cellular membrane. ZO-1, zonula occludens-1.

has a significant role in preventing bacterial translocation by altering pathogenetic mechanisms (16). Alcohol-induced intestinal barrier dysfunction may lead to increased intestinal permeability (17). However, the specific action sites at which alcohol may increase intestinal epithelial barrier permeability are not fully understood.

The Caco-2 cell line, originally derived from a human colon adenocarcinoma, resembles small intestinal enterocytes and spontaneously undergoes typical enterocytic differentiation (18). The Caco-2 monolayer cells exhibit a well-differentiated brush border containing tight junctions on the apical surface and have been extensively used to study intestinal epithelial barrier functions (19). The present study used an alcohol-treated Caco-2 intestinal epithelial cell monolayer in vitro model to investigate whether alcohol is able to induce intestinal barrier dysfunction and decrease expression of the tight junction-associated proteins, ZO-1 and claudin-1. The effects of alcohol on Caco-2 cell viability were assessed using the MTT assay, and the LDH concentration in supernatant fluid was assessed at $450 \mathrm{~nm}$ using a microplate reader. The MTT assay indicated that cell viability was not altered at alcohol concentrations of $<5 \%$, while concentrations of $\geq 7.5 \%$ led to a $\geq 20 \%$ reduction in Caco-2 cell viability. However, alcohol concentrations of $1-10 \%$ had virtually no impact on the LDH concentration in the supernatant fluid, and the Caco-2 cell membrane showed no evidence of morphological damage. Based on these results, an alcohol concentration of $5 \%$ was defined as the maximum concentration at which ethanol has no significant effect on Caco-2 cell viability and the molecular structure of tight junctions.

Smaller molecules penetrate enterocytes through a transcellular route via a large number of transmembrane channels and carriers, whereas larger molecules are transported across enterocytes through a paracellular pathway via intercellular tight junctions $(20,21)$. The permeability of the paracellular pathway is the molecular basis of the substance selectivity of epithelial cells (22). In the present study, the TEER and fluorescent yellow flux rate were utilized to estimate the effects of alcohol on the paracellular permeability of the Caco-2 cell monolayers. The flow of ions in the paracellular gap was represented by the TEER values. The results showed that alcohol induced a significant time-dependent decrease in the TEER, with the lowest value obtained at $60 \mathrm{~min}$ of alcohol treatment. A significant correlation was present between the decrease in the TEER value and the increase in the fluorescent yellow flux rate. A time-dependent increase in the fluorescent 
yellow flux rate as a result of the alcohol treatment was also observed. An increase in the TEER value and fluorescent yellow transmittance rate was observed within $60 \mathrm{~min}$ of incubation, indicating that the effects of alcohol on the paracellular permeability in the Caco-2 cell monolayers may be a transient process. Furthermore, western blot analysis was performed to detect the effects of alcohol on the expression of the tight junction-associated proteins, ZO-1 and claudin-1. ZO-1, also known as tight junction protein 1 , is an important member of the membrane-associated guanylate kinase homologs (MAGUK) family, having binding domains to adherens, tight junction proteins and the actin cytoskeleton (23). Claudin-1, a protein responsible for controlling cell-to-cell adhesion, is an integral component of tight junctions, regulating the paracellular and transcellular transport of solutes across human epithelia and endothelia (24). The present study demonstrated that ZO-1 expression exhibited a progressive decline at $20 \mathrm{~min}$ and reached its minimum level at $60 \mathrm{~min}$, with an increasing trend observed following $60 \mathrm{~min}$ of incubation, and claudin-1 expression showed an inverse correlation. Alterations in the expression of the ZO-1 and claudin-1 proteins showed consistent trends with changes in the TEER value and the fluorescent yellow transmittance rate in the Caco- 2 cells, indicating that ZO-1 and claudin-1 have important roles in alcohol-induced intestinal barrier dysfunction.

In conclusion, the results of the present study indicated that alcohol increases the permeability of the intestinal epithelial barrier in a dose- and time-dependent manner. Alcohol induces a change in the expression of the tight junction-associated proteins, ZO-1 and claudin-1, which are two major sites of alcohol action, thus increasing intestinal epithelial barrier permeability.

\section{Acknowledgements}

This study was funded by the Scientific Research of the First Hospital of China Medical University (fsfh1313).

\section{Referenes}

1. Bataller R and Gao B: Dissecting the role of CB1 receptors on chronic liver diseases. Gut 62: 957-958, 2013.

2. Li YM, Fan JG, Wang BY, et al: Guidelines for the diagnosis and management of alcoholic liver disease: update 2010: (published in Chinese on Chinese Journal of Hepatology 2010; 18: 167-170). J Dig Dis 12: 45-50, 2011.

3. Rao RK, Seth A and Sheth P: Recent Advances in Alcoholic Liver Disease I. Role of intestinal permeability and endotoxemia in alcoholic liver disease. Am J Physiol Gastrointest Liver Physiol 286: G881-G884, 2004.

4. Brun P, Castagliuolo I, Di Leo V, et al: Increased intestinal permeability in obese mice: new evidence in the pathogenesis of nonalcoholic steatohepatitis. Am J Physiol Gastrointest Liver Physiol 292: G518-G525, 2007.
5. Purohit V, Bode JC, Bode C, et al: Alcohol, intestinal bacterial growth, intestinal permeability to endotoxin, and medical consequences: summary of a symposium. Alcohol 42: 349-361, 2008.

6. Parlesak A, Schäfer C, Schütz T, Bode JC and Bode C: Increased intestinal permeability to macromolecules and endotoxemia in patients with chronic alcohol abuse in different stages of alcohol-induced liver disease. J Hepatol 32: 742-747, 2000.

7. Zima T and Kalousová M: Oxidative stress and signal transduction pathways in alcoholic liver disease. Alcohol Clin Exp Res 29 (11 Suppl): 110S-115S, 2005.

8. Turner JR: Intestinal mucosal barrier function in health and disease. Nat Rev Immunol 9: 799-809, 2009.

9. Yu J, Liu F, Yin P, et al: Involvement of oxidative stress and mitogen-activated protein kinase signaling pathways in heat stress-induced injury in the rat small intestine. Stress 16: 99-113, 2013.

10. Banan A, Keshavarzian A, Zhang L, et al: NF-kappaB activation as a key mechanism in ethanol-induced disruption of the F-actin cytoskeleton and monolayer barrier integrity in intestinal epithelium. Alcohol 41: 447-460, 2007.

11. Colgan SP and Taylor CT: Hypoxia: an alarm signal during intestinal inflammation. Nat Rev Gastroenterol Hepatol 7: 281-287, 2010.

12. Kong J, Zhang Z, Musch MW, et al: Novel role of the vitamin $D$ receptor in maintaining the integrity of the intestinal mucosal barrier. Am J Physiol Gastrointest Liver Physiol 294: G208-G216, 2008.

13. Ulluwishewa D, Anderson RC, McNabb WC, Moughan PJ, Wells JM and Roy NC: Regulation of tight junction permeability by intestinal bacteria and dietary components. J Nutr 141: 769-776, 2011.

14. Blaskewicz CD, Pudney J and Anderson DJ: Structure and function of intercellular junctions in human cervical and vaginal mucosal epithelia. Biol Reprod 85: 97-104, 2011.

15. Groschwitz KR and Hogan SP: Intestinal barrier function: molecular regulation and disease pathogenesis. J Allergy Clin Immunol 124: 3-22, 2009.

16. Balzan S, de Almeida Quadros C, de Cleva R, Zilberstein B and Cecconello I: Bacterial translocation: overview of mechanisms and clinical impact. J Gastroenterol Hepatol 22: 464-471, 2007.

17. Marchiando AM, Graham WV and Turner JR: Epithelial barriers in homeostasis and disease. Annu Rev Pathol 5: 119-144, 2010.

18. Fogh J, Fogh JM and Orfeo T: One hundred and twenty-seven cultured human tumor cell lines producing tumors in nude mice. J Natl Cancer Inst 59: 221-226, 1977.

19. Fischer A, Gluth M, Weege F, et al: Glucocorticoids regulate barrier function and claudin expression in intestinal epithelial cells via MKP-1. Am J Physiol Gastrointest Liver Physiol 306: G218-G228, 2014.

20. Colegio OR, Van Itallie CM, McCrea HJ, Rahner C and Anderson JM: Claudins create charge-selective channels in the paracellular pathway between epithelial cells. Am J Physiol Cell Physiol 283: C142-C147, 2002.

21. Ding LA and Li JS: Gut in diseases: physiological elements and their clinical significance. World J Gastroenterol 9: 2385-2389, 2003.

22. Muto S, Hata M, Taniguchi J, et al: Claudin-2-deficient mice are defective in the leaky and cation-selective paracellular permeability properties of renal proximal tubules. Proc Natl Acad Sci USA 107: 8011-8016, 2010.

23. Sobarzo CM, Lustig L, Ponzio R, Suescun MO and Denduchis B: Effects of di(2-ethylhexyl) phthalate on gap and tight junction protein expression in the testis of prepubertal rats. Microsc Res Tech 72: 868-877, 2009.

24. Krämer F, White K, Kubbies M, Swisshelm K and Weber BH: Genomic organization of claudin-1 and its assessment in hereditary and sporadic breast cancer. Hum Genet 107: 249-256, 2000. 